Marquette University

e-Publications@Marquette

3-1-2011

\title{
Association Between the Female Athlete Triad and Endothelial Dysfunction in Dancers
}

Anne Z. Hoch

Medical College of Wisconsin

Paula Papanek

Marquette University, paula.papanek@marquette.edu

Aniko Szabo

Medical College of Wisconsin

Michael E. Widlansky

Medical College of Wisconsin

Jane E. Schimke

Medical College of Wisconsin

See next page for additional authors

Accepted version. Clinical Journal of Sport Medicine, Vol. 21, No. 2 (March 2011). DOI: 10.1097/ JSM.0b013e3182042a9a. C 2011 Lippincott Williams \& Wilkins, Inc. Used with permission. 
Authors

Anne Z. Hoch, Paula Papanek, Aniko Szabo, Michael E. Widlansky, Jane E. Schimke, and David D. Gutterman 


\title{
Association between the Female Athlete Triad and Endothelial Dysfunction in Dancers
}

Authors: Anne Z. Hoch, DO, Paula Papanek, PhD, MPT, LAT, Aniko Szabo, PhD, Michael E. Widlansky, MD, Jane E. Schimke, AAS, and David D. Gutterman, MD

\begin{abstract}
:
Objective: To determine the prevalence of the 3 components of the female athlete triad [disordered eating, menstrual dysfunction, low bone mineral density (BMD)] and their relationships with brachial artery flow-mediated dilation in professional dancers.

Design: Prospective study.

Setting: Academic institution in the Midwest.

Participants: Twenty-two professional ballet dancers volunteered for this study.

Interventions: The prevalence of the female athlete triad and its relationship to endothelial dysfunction.
\end{abstract}

Main Outcome Measures: Subjects completed questionnaires to assess disordered eating and menstrual status/history. They also completed a 3-day food record and wore an accelerometer for 3 days to determine energy availability. Serum baseline thyrotropin, prolactin, and hormonal concentrations were obtained. Bone mineral density and body composition were measured with a GE Lunar Prodigy dual-energy X-ray absorptiometry. Endothelial function was determined as flow-mediated vasodilation measured by high-frequency ultrasound in the brachial artery. An increase in brachial diameter $<5 \%$ to hyperemic flow stimulus was defined a priori as endothelial dysfunction.

Results: Seventeen dancers (77\%) had evidence of low/negative energy availability. Thirty-two percent had disordered eating (EDE-Q score). Thirty-six percent had menstrual dysfunction and 14\% were currently using hormone contraception. Twenty-three percent had evidence of low bone density (Z-score < -1.0). Sixty-four percent had abnormal brachial artery flow-mediated dilation (<5\%). Flow-mediated dilation values were significantly correlated with serum estrogen and whole-body and lumbar BMD. All the 3 components of the triad plus endothelial dysfunction were present in $14 \%$ of the subjects.

Conclusions: Endothelial dysfunction was correlated with reduced BMD, menstrual dysfunction, and low serum estrogen. These findings may have profound implications for cardiovascular and bone health in professional women dancers. 


\section{Introduction}

There are an estimated 38,000 professional dancers in the United States and countless amateur or recreational dancers. This unique group of athletes is at high risk for developing the female athlete triad (triad) $)^{1,2}$ due to the aesthetic nature of the sport. ${ }^{3}$ The first American College of Sports Medicine triad position statement was published in 1997 describing the interrelated conditions of disordered eating, amenorrhea of hypothalamic origin, and osteoporosis. In 2007, the American College of Sports Medicine revised their position statement on the triad, including updated recommendations for screening, diagnosis, prevention, and treatment. One new aspect of the position statement is a comprehensive and expanded definition of each component of the triad from a spectrum of health to disease, including energy availability (EA) (with or without disordered eating), menstrual function, and bone health. ${ }^{1}$ Low/negative EA has been determined to be the key disorder underlying the relationship to the other components. ${ }^{1,4}$

The new position statement also includes seminal research from our group that demonstrated an association between athletic amenorrhea and a reduction in brachial artery flow-mediated dilation (FMD). ${ }^{1,5}$ Abnormal brachial dilation has been shown to have a positive predictive value (95\%) for coronary endothelial dysfunction. ${ }^{6}$ In addition, Schachinger et al $^{7}$ have shown that coronary endothelial vasodilator dysfunction predicts long-term atherosclerotic disease progression and cardiovascular event rates. Therefore, the assessment of brachial endothelial function can provide valuable information about cardiovascular disease risk in an otherwise healthy athletic population.

No studies have examined the relationship of all the 3 components of the triad while simultaneously assessing brachial artery FMD in professional dancers, a high-risk group for the triad. Therefore, the purpose of this study is to determine the prevalence of the 3 components of the triad [disordered eating (with/without low/negative EA), menstrual dysfunction, low bone mineral density (BMD)] and their relationships with brachial artery FMD.

\section{Methods}

\section{Subjects and Experimental Design}

This study was reviewed and approved by multi-institutional human subjects' internal review boards (Medical College of Wisconsin, Marquette University, Milwaukee, Wisconsin). Inclusion criteria included professional female dancers, aged 18 to 35 years, from a single company in the Midwest. Exclusion criteria included a history of heart disease, vascular disease, hypertension, diabetes, hypercholesterolemia, chromosomal disorder, current pregnancy, pituitary tumor, anomalies of the reproductive system, and thyroid disease. Subjects on a cardiac, 
cholesterol, hypertension, or thyroid medication were excluded. Subjects were not excluded based on ethnicity. Twenty-two women met the inclusion criteria and completed the study. No women were excluded based on our criteria. The study was explained in person, and an informed consent was obtained. Medical histories were obtained by questionnaires and interview.

\section{Disordered Eating}

Energy availability is the amount of dietary energy remaining for other bodily functions after exercise energy expenditure ${ }^{1,4}$ and has been demonstrated to be the key in terms of initiating the neuroendocrine cascade associated with the triad. ${ }^{1,4}$ Energy intake was determined from a prospective 3-day food diary recorded during 2 weekdays and 1 weekend day during the training season. A registered research dietitian met with each dancer for education and training in proportions or size of food servings, proper recording of foods, and the use of the food diary in general. Dietary analysis was performed by the research dietician using Nutrition Data System for Research software version 2007 (developed by the Nutrition Coordinating Center, University of Minnesota, Minneapolis, Minnesota) to determine total calories, total composition of fat, protein, carbohydrates, and micronutrient content. Subjects were encouraged to weigh their food portions for recording.

Estimated energy expenditure during daily exercise was determined by use of an Actigraph accelerometer (Actigraph GT1M Monitor; Actigraph, Pensacola, Florida). Accelerometers were individually calibrated for each subject at 2 different self-selected intensities of exercise with the Cortex metabolic system (Cortex MetaLyzer 3B Version 2.1; Cortex, Leipzig, Germany). Accelerometers were worn continuously for 72 hours, and the total kilocalories expended were calculated. Accelerometer has been shown to be a valid device to measure energy expenditure. .,9 $^{2,}$

Energy availability was calculated from the subjects' caloric intake minus the amount of exercise energy expended over the same time as determined from the accelerometer.

Low/negative EA was defined as a negative value. At the end of the study, each subject met with the dietician to review her results.

Eating habits and attitudes were assessed by the eating disorder examination questionnaire (EDE-Q). The EDE-Q is a shorter version of the eating disorder examination ${ }^{10}$ and is designed for self-report. The EDE-Q is composed of 4 scales [weight concern, shape concern, eating concern, dietary restraint] and has a global score or mean composite score of the 4 subscales. Scores ranging from 0 to 6 on a Likert scale correspond to the number of days the 
respondent has experienced a specific attitude, feeling, or behavior during the past 4 weeks. Athletes were classified as having disordered eating if they had a mean score of $\geq 4.0$ on any subscale. A cutoff score of 4.0 indicates that a specific attitude or behavior was reported on more than half of the past 28 days, which was used to define disordered eating because it has been shown to be predictive of eating disorders. ${ }^{10}$ Previously, scores of $\geq 4.0$ have been reported to correspond to the 80th to 95th percentiles from reference norms for adolescent girls. ${ }^{10}$ Based on this scoring rubric, athletes were divided into 2 groups: disordered eating (elevated EDE-Q score) or normal EDE-Q score.

\section{Menstrual Status}

Self-administered questionnaires were used to determine age of menarche, number of periods per year, number of days between periods, skipped periods, history of thyroid disease or pituitary tumor, and use and type of birth control. A formal interview regarding menstrual status and medical history was conducted by the principal investigator. For the purpose of this study, eumenorrhea or regular cycling was defined as menstrual bleeding every 28 to 30 days. Primary amenorrhea was defined as the absence of menarche by age 15 and secondary amenorrhea as the cessation of menses for $>3$ consecutive cycles after onset of menarche. Oligomenorrhea was represented by menstrual cycles with intervals $>35$ days.

\section{Hormonal Profiles}

To determine the role of reproductive and metabolic hormones and to rule out potential medical or gynecological causes of amenorrhea, follicle-stimulating hormone, luteinizing hormone, progesterone, estrogen, thyrotropin, prolactin, and beta-human chorionic gonadotropin were measured. Hormone concentrations were measured in plasma obtained from whole venous blood drawn in the early follicular phase of the menstrual cycle (days 4-8) in the early morning after an 8-hour fast. Amenorrheic dancers followed the same protocol except, since they were not having menstrual cycles, they were tested at a convenient time. Samples were processed within hours and kept on ice until transported to the laboratory for analysis. All measurements were made in a clinical certified laboratory (College of American Pathologists and Clinical Laboratory Improvement Amendments).

\section{Bone Mineral Density}

Each subject underwent BMD measurement by dual-energy X-ray absorptiometry using a GE Lunar Prodigy densitometer, version 7.52.002 (Madison, Wisconsin). Calibrations with a 
standard phantom spine were performed daily. All measurements were performed by the same trained professional. The coefficient of variation of the phantom for more than a 6-month period was $0.29 \%$. Bone mineral density (gram/square centimeter) of the lumbar spine (L1-L4), total left hip, left femoral neck, left trochanter, and total body were measured for each subject.

\section{Cardiovascular Status}

Brachial artery FMD and velocity were determined by techniques previously used in this laboratory. ${ }^{5}$ Subjects were studied in a supine resting position after an 8-hour fast in a temperature-controlled room. Systemic arterial pressure was periodically monitored in the nondominant arm with an automated sphygmomanometer. Subjects were instrumented for continuous recording of electrocardiogram, heart rate, and intermittent blood pressure. All subjects were tested at approximately the same time of day with eumenorrheic subjects studied in the early follicular phase of the menstrual cycle (days 4-8). Amenorrheic subjects were tested randomly as scheduled but also in the early morning.

\section{Statistics}

The relationship between menstrual status (eumenorrhea, oligomenorrhea, and amenorrhea) and FMD was evaluated using the nonparametric Jonckheere-Terpstra exact test for trend after excluding 3 subjects using hormonal birth control. The relationship between estrogen concentrations and FMD was assessed using Spearman rank correlation for the same population with an additional exclusion of 1 ovulating subject. Pearson correlation coefficient was used to determine a correlation among FMD, BMD, and EA.

\section{Results}

\section{Subject Characteristics}

Twenty-two elite dancers were consented and completed all measurements in this study. Dancers self-reported their ethnicity as white $(n=20)$, Asian $(n=1)$, and Hispanic $(n=1)$, with a mean $( \pm S D)$ age of $23.2 \pm 4.7$ years. Table 1 provides descriptive demographics of the cohort.

\section{Energy Availability/Disordered Eating}

Seventeen dancers (77\%) had low/negative EA (-547.8 $\pm 359.9 \mathrm{kcal} / \mathrm{d})$ (Figure 1A). Seven dancers $(32 \%)$ had elevated EDE-Q scores of $>4.0$ in one or more subcategories, accounting for a total of 14 elevated scores. Eighty-six percent either had abnormal EDE-Q scores and/or low/negative EA. Thirteen dancers (59\%) were in a calcium deficit $(-441.3 \pm 347.7$ 
$\mathrm{mg} / \mathrm{d}$ ) based on a requirement of $1500 \mathrm{mg} / \mathrm{d} .{ }^{11}$ Finally, 10 (46\%) were in an iron deficit (mean deficit of $5.5 \mathrm{mg} / \mathrm{d}$ ) based on a requirement of $18 \mathrm{mg} / \mathrm{d}$.

\section{Amenorrhea/Oligomenorrhea}

Current menstrual status is reported in Figure 1B. Most of the dancers self-reported a history of menstrual dysfunction. Four (18\%) had a history of primary amenorrhea, 14 (64\%) had a history of secondary amenorrhea, and $6(27 \%)$ reported a history of oligomenorrhea. At the time of the study, 2 dancers were amenorrheic (9\%) $(2.0 \pm 0.1$ years) and $6(27 \%)$ were oligomenorrheic. Collectively, 36\% self-reported current menstrual dysfunction. Three (14\%) were taking different forms of combination contraceptive hormones. Beta-human chorionic gonadotropin tests results were negative. Thyrotropin and prolactin concentrations were within normal limits.

\section{Bone Mineral Density}

The American College of Sports Medicine/World Health Organization (Z-score < -1.0) criteria are reported (Figure $1 C)^{1,12}$ Five dancers (23\%) had low BMD in 1 or more locations. International Society for Clinical Densitometry (Z-score < -2.0) criteria were not met by any of our dancers in any anatomic locations studied. ${ }^{13}$ Five $(23 \%)$ had low BMD in the lumbar spine (L2-L4), 2 (9\%) had low whole-body BMD and lumbar spine, and no dancers demonstrated low BMD in either the left femoral trochanter or left femoral neck. Collectively, $23 \%$ had a clinically significant decrease in BMD at least at 1 site as determined by the dual-energy $\mathrm{X}$-ray absorptiometry.

\section{Vascular Reactivity}

Brachial artery data are summarized in Table 2 and Figure 2. Table 2 includes data on heart rate, systolic arterial pressure, diastolic arterial pressure, temperature, relative humidity, baseline brachial artery diameter, FMD, and peak change in flow velocity. Subjects were subsequently divided into normal and reduced FMD as defined by $<5 \%$ dilation in response to an ischemic challenge ${ }^{14,15}$ and unpublished norms for our laboratory. Brachial artery FMD was reduced in 14 dancers (64\%). Baseline brachial diameters, arterial pressures, resting heart rate, temperature, humidity, and peak change in flow velocity were not significantly different between subjects with reduced and with normal FMD.

All subjects with current menstrual dysfunction $(n=8 ; 36 \%)$ had a reduced FMD. A significant trend was found between menstrual status and FMD (Figure 3A). Amenorrheic 
dancers $(n=2)$ had a mean FMD of $1.8 \% \pm 0.4$. Oligomenorrheic dancers $(n=6)$ had a mean FMD of $3.1 \% \pm 1.7 \%$. Five of the eumenorrheic dancers also had a reduced FMD $(3.0 \% \pm 1.8 \%)$. A significant difference was found $(P=0.016)$ for FMD between the amenorrheic group $(n=2)$ and total eumenorrheic group $(n=11)(1.8 \% \pm 0.4 \%$ vs $5.9 \% \pm 3.0 \%)$. Furthermore, lower estrogen values were associated with significantly lower FMD $(P=0.026)$ (Figure 3B). Subjects with the lowest Z-score also had the lowest FMD. The strongest positive correlation $(R=0.50 ; P$ $=0.02$ ) was between FMD and whole-body Z-score (Figures 3C, D). L2 to L4 Z-score also correlated to FMD $(R=0.46 ; P=0.03)$ (Figure $3 C)$. Interestingly, there was no correlation between FMD and EA (Figure 3E).

\section{Prevalence of Triad Components}

In the present study of elite dancers, $3(14 \%)$ had all the 3 components of the triad and reduced flow-mediated vasodilation. An additional 5 (23\%) had 3 of the 4 components. Three $(14 \%)$ had 2 components, $8(36 \%)$ had at least 1 component, and $3(14 \%)$ did not have a single component.

\section{Discussion}

This is the first study to report on the prevalence of all the 3 components of the triad plus the emerging component of endothelial dysfunction manifested by reduced brachial artery FMD in professional ballet dancers. The percentage of dancers who met all the 3 components of the triad and reduced FMD was 14\% (3 of 22) with an additional 23\% (5 of 22) meeting the criteria for 3 of the 4 components. This is much higher than that in the study by Torstveit and Sundgot-Borgen ${ }^{16}$ of elite Norwegian endurance athletes, where only $4.3 \%(N=8)$ met the criteria for 3 components of the triad. Although none of those athletes were professional dancers, 6 competed in leanness type sports (running, swimming, biathlon, and bicycling). The prevalence in our population is also much higher than leanness sports (track, cross-country, and swimming) ${ }^{18}$ To previously reported in high school athletes $(1 \%)^{17,18}$ from date, the majority of studies on dancers have looked primarily a variety of sports in which $62 \%(\mathrm{~N}=106)$ competed in at the disordered eating or the link between amenorrhea and low BMD, ${ }^{19-21}$ and none have investigated the prevalence of endothelial dysfunction. Of the studies specifically designed to determine the prevalence of all the 3 components of the triad simultaneously, the majority have focused on elite Norwegian endurance athletes, ${ }^{16,22}$ college athletes, ${ }^{23}$ or high school athletes, ${ }^{17,18}$ across a spectrum of sports. The prevalence rate of $14 \%$ for the triad plus reduced FMD in elite dancers suggests that the changes in vascular function coexist in sufficient 
frequency that a tetrad of components better describes these athletes.

\section{Flow-Mediated Dilation}

No studies have investigated the prevalence of reduced FMD in professional dancers, a group known to be at high risk for athletic amenorrhea. ${ }^{21,24}$ Previously, we reported reduced brachial artery FMD in collegiate runners with amenorrhea (mean FMD, $1.08 \% \pm 0.91$ ). ${ }^{5}$ Similarly, Rickenlund et $\mathrm{al}^{25}$ reported a reduced mean FMD of $1.1 \%$ in endurance athletes from Sweden. These results are similar to our amenorrheic $(n=2$; mean FMD, $1.8 \% \pm 0.4)$ and oligomenorrheic dancers $(n=6$; mean FMD, $3.1 \% \pm 1.7)$ in this study.

The decrease in reactive hyperemic response, although consistent with our previous work in runners, is surprising. Classically, exercise training enhances endothelium-dependent vasodilation in peripheral arteries. Elite dancers use both upper and lower extremities for prolonged periods. However, previous studies ${ }^{26}$ have shown that elite dancers have a $\dot{V} o_{2} \max$ in the range of nonendurance athletes. Therefore, the decreased FMD response may be partially due to suboptimal aerobic fitness. Future studies should measure oxygen consumption directly to better evaluate this variable.

This study represented a sample of women displaying heterogenous hormonal disturbances and 3 subjects taking hormone contraceptives. However, all amenorrheic and oligomenorrheic dancers in this study (8 of 22) had low FMD. Low estrogen concentrations were correlated with low FMD. However, 5 of the 11 eumenorrheic and the 2 dancers using hormone contraception also had a reduced FMD, indicating that FMD is not simply a function of circulating estrogen concentrations. Furthermore, of the 17 subjects with low/negative EA (-547.8 \pm 359.9 $\mathrm{kcal} / \mathrm{d}), 71 \%$ demonstrated reduced FMD.

These data suggest that endothelial dysfunction is a complex process, which is not solely determined by estrogen concentrations. It seems that the regulation of flow-mediated vasodilation, a direct index of endothelial function, may be an integration of menstrual status, and thus hormone concentrations, aerobic fitness, and EA. Additional nutrient differences may also play a role but have yet to be investigated. The relationship between menstrual status/estrogen concentrations and FMD is statistically and clinically relevant. Not only is amenorrhea associated with reduced BMD but it is also associated with reduced FMD and potentially an increased risk for cardiovascular disease in otherwise healthy young women.

Three dancers had all 4 components of what we suggest is a tetrad of coexisting conditions. This is much higher than other studies examining only 3 components of the triad. ${ }^{16-18}$ We hypothesize that vascular endothelial function is modulated by dietary nutrient content, EA 
(both intake and expenditure), estrogen concentrations, and aerobic condition. The ability to appropriately vasodilate in response to flow is vital not only for facilitating athletic performance and recovery but also in terms of vascular health. The high incidence of abnormal brachial artery FMD is alarming, and additional work is required to determine whether these changes are predictive of future cardiac and vascular disease because they are in other populations. ${ }^{27}$ Further studies are needed to ascertain the relationship of decreased FMD with cardiac function and human performance as well as underlying mechanisms.

There are several limitations to this study. The sample size is very small, making multiple comparisons unachievable. However, the major methodological flaw in this study is pooling statistical analyses of athletes using hormonal contraceptives together with those who are not. Furthermore, the authors had access to 1 professional dance company, making it difficult to determine true prevalence. In addition, we had no control group. However, finding age-matched and body mass index-matched controls proved to be unattainable. Finally, $\dot{\mathrm{V}}_{2}$ testing would be valuable to measure because this is a known determinant of FMD.

\section{Conclusions}

Our data show that the prevalence of the 4 components (triad and reduced endothelial function) is comparably high in professional dancers (14\%). Abnormal FMD occurred in $64 \%$ of our population. Low FMD was associated with low estrogen concentrations and reduced BMD. Collectively, this suggests that it is not simply a function of circulating plasma estrogen concentrations but is more complex and multifactorial. Although additional studies are required to determine mechanisms, the high incidence of low/negative EA, BMD, and menstrual dysfunction accentuates target interventions to address this issue in the dance community and raises concern regarding long-term impact on the vascular system.

\section{Acknowledgments}

The authors thank Michael Pink and the Milwaukee Ballet Company for participating in this study.

\section{References}

1. Nattiv A, Loucks AB, Manore MM, et al. American College of Sports Medicine position stand. The female athlete triad. Med Sci Sports Exerc. 2007;39:1867-1882.

2. Otis CL, Drinkwater B, Johnson M, et al. American College of Sports Medicine position 
stand. The female athlete triad. Med Sci Sports Exerc. 1997;29:1669-1671.

3. Torstveit MK, Sundgot-Borgen J. Participation in leanness sports but not training volume is associated with menstrual dysfunction: a national survey of 1276 elite athletes and controls. Br J Sports Med. 2005;39:141-147.

4. Loucks AB, Verdun M, Heath EM. Low energy availability, not stress of exercise, alters LH pulsatility in exercising women. J Appl Physiol. 1998; 84:37-46.

5. Hoch AZ, Dempsey RL, Carrera GF, et al. Is there an association between athletic amenorrhea and endothelial cell dysfunction? Med Sci Sports Exerc. 2003;35:377-383.

6. Anderson TJ, Uehata A, Gerhard MD, et al. Close relation of endothelial function in the human coronary and peripheral circulations. J Am Coll Cardiol. 1995;26:1235-1241.

7. Schachinger V, Britten MB, Zeiher AM. Prognostic impact of coronary vasodilator dysfunction on adverse long-term outcome of coronary heart disease. Circulation. 2000;101:1899-1906.

8. Abel MG, Hannon JC, Sell K, et al. Validation of the Kenz Lifecorder EX and ActiGraph GT1M accelerometers for walking and running in adults. Appl Physiol Nutr Metab. 2008;33:1155-1164.

9. Herrmann S, Hart T, Lee C, et al. Evaluation of the MyWellness Key accelerometer. Br J Sports Med. 2011;45:109-113.

10. Carter JC, Stewart DA, Fairburn CG. Eating disorder examination questionnaire: norms for young adolescent girls. Behav Res Ther. 2001; 39:625-632.

11. Institute of Medicine. Dietary Reference Intake For Calcium, Phosphorus, Magnesium, Vitamin D and Fluoride. Washington, DC: National Academies Press; 1989.

12. Assessment of fracture risk and its application to screening for postmenopausal osteoporosis. Report of a WHO Study Group. World Health Organ Tech Rep Ser. 1994;843:1-129.

13. Writing Group for the ISCD Position Development Conference. Diagnosis of osteoporosis in men, premenopausal women, and children. J Clin Densitom. 2004;7:17-26.

14. Hoch AZ, Lynch SL, Jurva JW, et al. Folic acid supplementation improves vascular function in amenorrheic runners. Clin J Sport Med. 2010;20: 205-210.

15. Hoch AZ, Pajewski NM, Hoffmann RG, et al. Possible relationship of folic acid supplementation and improved flow-mediated dilation in premenopausal, eumenorrheic athletic women. J Sports Sci Med. 2009;8: 123-129.

16. Torstveit MK, Sundgot-Borgen J. The female athlete triad exists in both elite athletes and controls. Med Sci Sports Exerc. 2005;37:1449-1459. 
17. Hoch AZ, Pajewski NM, Moraski L, et al. Prevalence of the female athlete triad in high school athletes and sedentary students. Clin J Sport Med. 2009;19:421-428.

18. Nichols JF, Rauh MJ, Lawson MJ, et al. Prevalence of the female athlete triad syndrome among high school athletes. Arch Pediatr Adolesc Med. 2006;160:137-142.

19. Ringham $R$, Klump K, Kaye W, et al. Eating disorder symptomatology among ballet dancers. Int J Eat Disord. 2006;39:503-508.

20. Thomas JJ, Keel PK, Heatherton TF. Disordered eating attitudes and behaviors in ballet students: examination of environmental and individual risk factors. Int J Eat Disord. 2005;38:263-268.

21. Warren MP, Brooks-Gunn J, Fox RP, et al. Osteopenia in exercise-associated amenorrhea using ballet dancers as a model: a longitudinal study. J Clin Endocrinol Metab. 2002;87:3162-3168.

22. Torstveit MK, Sundgot-Borgen J. The female athlete triad: are elite athletes at increased risk? Med Sci Sports Exerc. 2005;37:184-193.

23. Beals KA, Hill AK. The prevalence of disordered eating, menstrual dysfunction, and low bone mineral density among US collegiate athletes. Int J Sport Nutr Exerc Metab. 2006;16:1-23.

24. Fogelholm M, Van Marken LW, Ottenheijm R, et al. Amenorrhea in ballet dancers in the Netherlands. Med Sci Sports Exerc. 1996;28:545-550.

25. Rickenlund A, Eriksson MJ, Schenck-Gustafsson K, et al. Amenorrhea in female athletes is associated with endothelial dysfunction and unfavorable lipid profile. $J$ Clin Endocrinol Metab. 2005;90:1354-1359.

26. Cohen JL, Segal KR, Witriol I, et al. Cardiorespiratory responses to ballet exercise and the $\dot{V}_{2}$ max of elite ballet dancers. Med Sci Sports Exerc. 1982;14:212-217.

27. Maldonado FJ, Miralles JH, Aguilar EM, et al. Relationship between noninvasively measured endothelial function and peripheral arterial disease. Angiology. 2009;60:725731. 


\section{Appendix}

\section{Figure 1: Prevalence of the Female Athlete Triad}

A

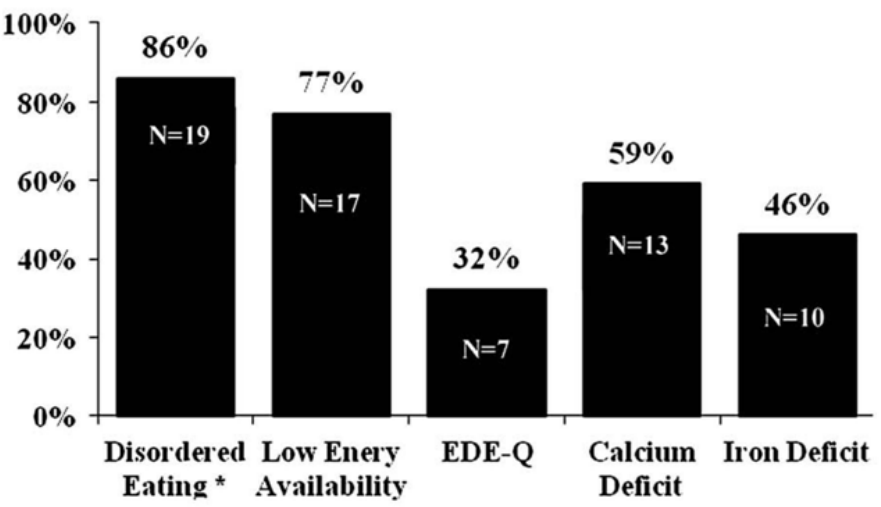

A. Percentage of dancers with disordered eating defined as calorie deficit or abnormal eating disorder examination questionnaire score. Calcium deficit based on $1500 \mathrm{mg}$ and iron deficit based on $18 \mathrm{mg}$ is also reported.

* Combination of low energy availability and/or abnormal EDE-Q

B

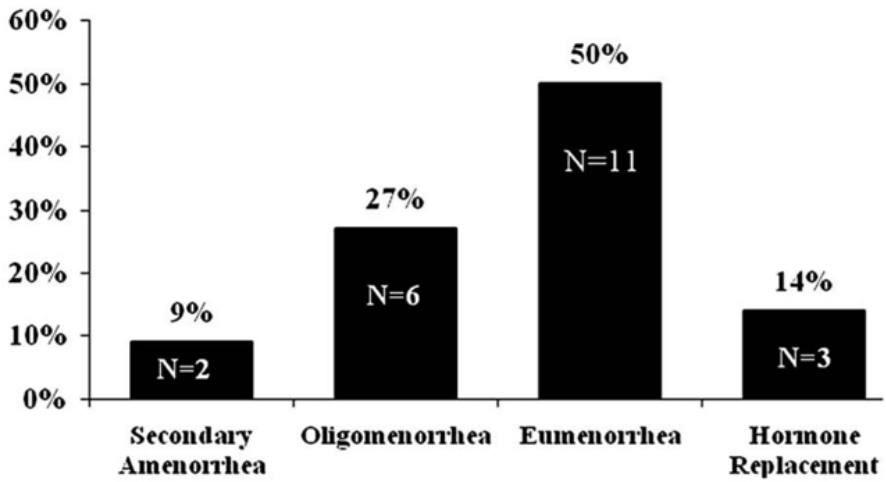

B. Current menstrual status based on questionnaire, interview, and blood work.

C

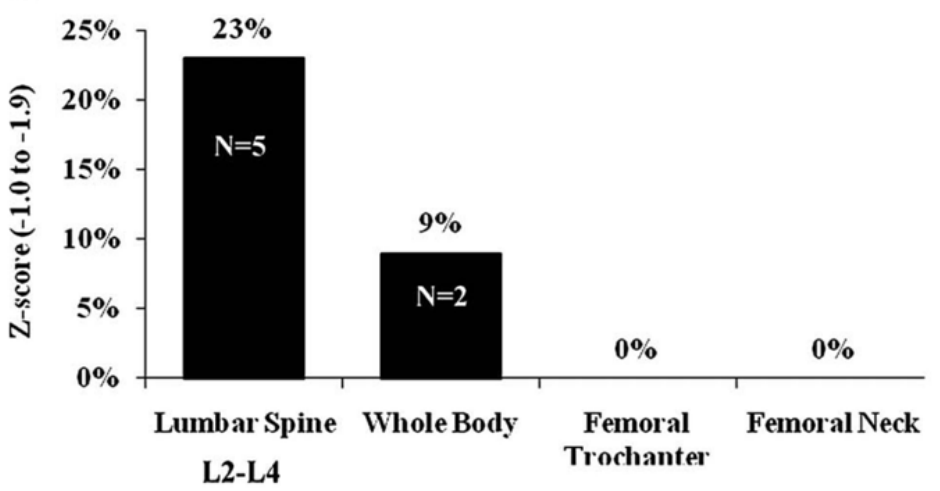

C. Bone mineral density in the dancers. A total of 5 subjects had reduced bone mineral density (BMD). Two subjects had reduced BMD in both the lumbar spine and whole body. 
Figure 2: Brachial Artery FMD Response to Ischemia

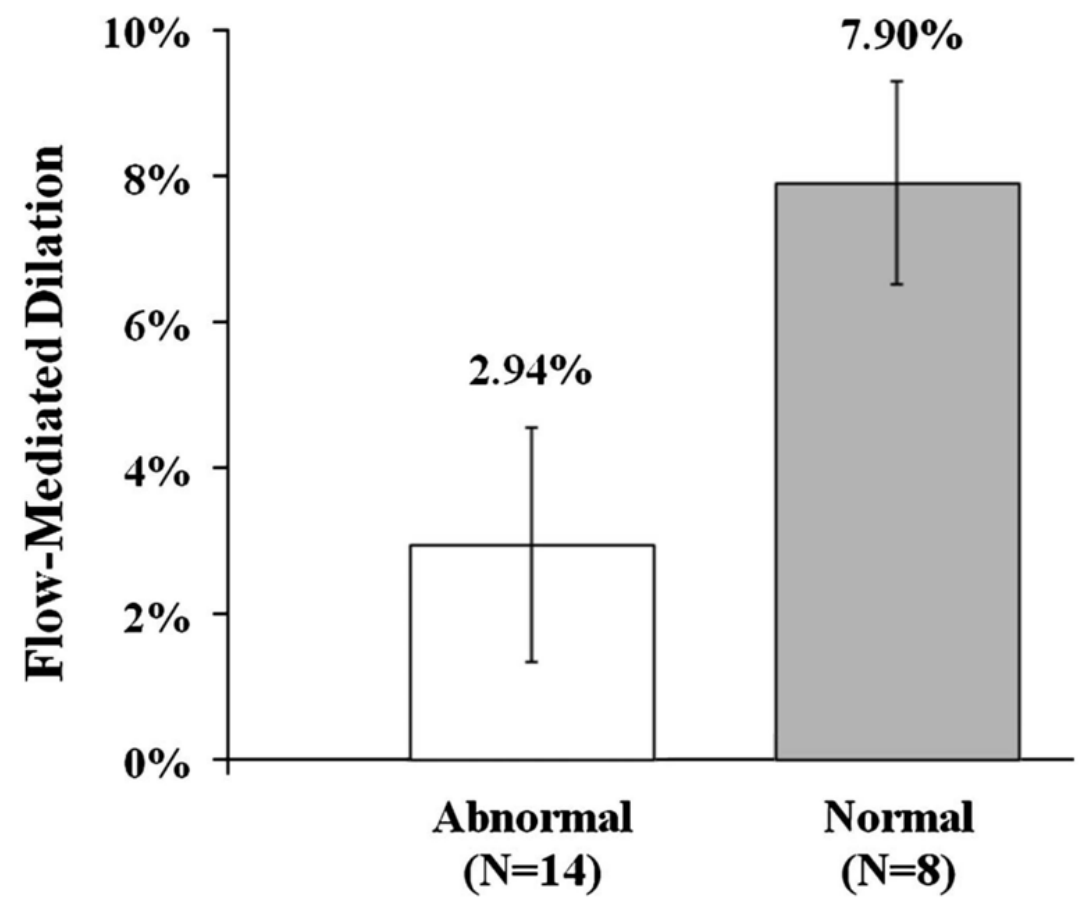

Fourteen dancers had reduced FMD (defined a priori as $<5 \%$ ), ${ }^{14,15}$ and 8 dancers had normal FMD. 
Figure 3: Correlations with Flow-Mediated Dilation (FMD)
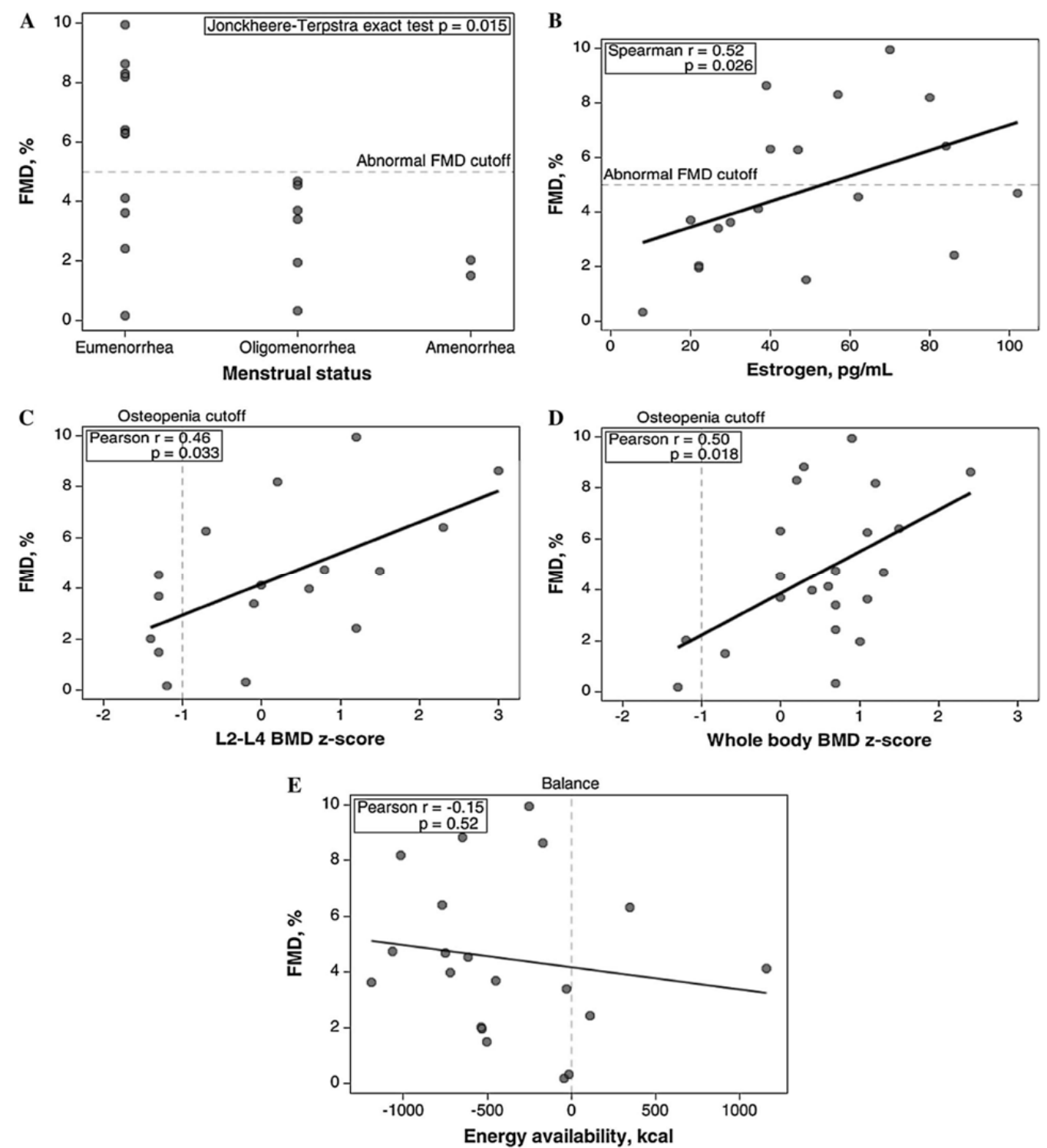

A. Relationship between menstrual status and FMD. All the subjects with oligomenorrhea and amenorrhea had abnormal FMD $(P=0.015)$.

B. Correlation between estrogen concentration (pictogram/milliliter) and FMD. A low estrogen concentration correlated with a low FMD $(P=0.026)$.

C. Correlation between lumbar spine (L2-L4) bone mineral density (BMD) and FMD. A low BMD Z-score correlated with a low FMD $(P=0.033)$.

D. Correlation between whole-body Z-score and FMD. A low BMD Z-score correlated with a low FMD $(P=$ 0.018).

E. Correlation between energy expenditure (kilocalories) and FMD. There was no correlation between energy expenditure and FMD $(P=0.52)$. 


\section{Table 1: Physical Characteristics}

\begin{tabular}{lc}
\hline \multicolumn{1}{c}{ Characteristic } & Ballet Dancers $(\mathbf{N}=\mathbf{2 2})$ \\
\hline Age, $\mathrm{y}$ & $23.2 \pm 4.7$ \\
Height, in & $64.8 \pm 2.8$ \\
Weight, lb & $114.8 \pm 10.3$ \\
Body fat, \% & $16.7 \pm 4.9$ \\
Body mass index, $\mathrm{kg} / \mathrm{m}^{2}$ & $19.29 \pm 1.1$ \\
Age of menarche, $\mathrm{y}$ & $13.6 \pm 1.8$ \\
Years of elite dancing & $17.0 \pm 4.5$ \\
\hline
\end{tabular}

Values are mean \pm SD unless otherwise indicated. 


\section{Table 2: Brachial Artery Studies}

\begin{tabular}{lccc}
\hline \multicolumn{1}{c}{ Ballet Dancers } & $\begin{array}{c}\text { Abnormal FMD, } \\
<\mathbf{5 \%}(\mathbf{n}=\mathbf{1 4})\end{array}$ & $\begin{array}{c}\text { Normal FMD, } \\
\mathbf{2 5 \%}(\mathbf{n}=\mathbf{8})\end{array}$ & $\boldsymbol{P}$ \\
\hline Heart rate, beats/min & $56.1 \pm 9.9$ & $54.9 \pm 8.8$ & 0.78 \\
Arterial pressure, mm Hg & $101.2 \pm 10.0$ & $99.0 \pm 5.3$ & 0.57 \\
$\quad$ Systolic & $60.6 \pm 8.9$ & $62.1 \pm 4.6$ & 0.67 \\
$\quad$ Diastolic & $69.3 \pm 1.3$ & $69.9 \pm 0.6$ & 0.25 \\
Temperature, ${ }^{\circ} \mathrm{F}$ & $21.2 \pm 4.5$ & $20.0 \pm 0.0$ & 1.0 \\
$\begin{array}{l}\text { Relative humidity, \% } \\
\text { Baseline brachial artery }\end{array} \quad 2.8 \pm 0.3$ & $2.6 \pm 0.4$ & 0.21 \\
$\quad$ diameter, mm & $2.9 \pm 0.4$ & $2.8 \pm 0.4$ & 0.67 \\
$\begin{array}{l}\text { Peak brachial artery } \\
\text { diameter, mm }\end{array}$ & $2.9 \pm 1.6$ & $7.9 \pm 1.4$ & - \\
FMD, \% & $67.2 \pm 41.7$ & $75.2 \pm 30.3$ & 0.64 \\
Peak change in flow velocity, \% & & & \\
\hline
\end{tabular}

Values are mean \pm SD unless otherwise indicated.

FMD, flow-mediated dilation. 